\title{
DIRECTED POLYMERS IN RANDOM ENVIRONMENT WITH HEAVY TAILS
}

\author{
ANTONIO AUFFINGER AND OREN LOUIDOR
}

\begin{abstract}
We study the model of Directed Polymers in Random Environment in $1+1$ dimensions, where the distribution at a site has a tail which decays regularly polynomially with power $\alpha$, where $\alpha \in(0,2)$. After proper scaling of temperature $\beta^{-1}$, we show strong localization of the polymer to a favorable region in the environment where energy and entropy are best balanced. We prove that this region has a weak limit under linear scaling and identify the limiting distribution as an $(\alpha, \beta)$-indexed family of measures on Lipschitz curves lying inside the $45^{\circ}$-rotated square with unit diagonal. In particular, this shows order $n$ transversal fluctuations of the polymer. If, and only if, $\alpha$ is small enough, we find that there exists a random critical temperature below which, but not above, the effect of the environment is macroscopic. The results carry over to $d+1$ dimensions for $d>1$ with minor modifications.
\end{abstract}

\section{INTRODUCTION}

Originally introduced in [7], Directed Polymers in Random Environment is a model for an interaction between a polymer chain and a medium with microscopic impurities. The reader can find mathematical and physical background material in surveys [3] and [5]. In this model, the medium with defects or environment is represented by a random measure $\sigma$ with support in $\mathbb{Z}_{+} \times \mathbb{Z}^{d}$, the shape of the polymer (random due to thermal fluctuations) is represented by the $Z_{+} \times \mathbb{Z}^{d}$-trajectory of a simple random walk on $\mathbb{Z}^{d}$ and the interaction is expressed as a measure change. More precisely, conditioned on $\sigma$, the $n$-monomer polymer chain is a random nearest-neighbor path $s:[0, n] \cap \mathbb{Z} \rightarrow \mathbb{Z}^{d}$ chosen according to the following Gibbs distribution:

$$
\mu_{\beta}^{\sigma}(s)=\frac{1}{Q_{\beta}^{\sigma}} \exp \left(-\beta \mathcal{H}^{\sigma}(s)\right) \quad ; \quad \mathcal{H}^{\sigma}(s)=-\sigma(s)
$$

where $\mathcal{H}^{\sigma}(s)$ is the Hamiltonian or energy of $s, \sigma(s)$ is to be understood as the measure under $\sigma$ of $\operatorname{graph}(s)=\{(x, s(x)): x \in[0, n] \cap \mathbb{Z}\}$, the parameter $\beta \in[0, \infty)$ represents the overall strength of the interaction (inverse temperature) and $Q_{\beta}^{\sigma}$ is the normalizing constant.

It is believed [5] that there exist thermodynamic phases in which the effects of the environment on the shape of the polymer become macroscopic in scale. More explicitly, it is expected that in $d \leqslant 2$ and any finite temperature, or $d>2$ and low enough (but not necessarily zero, at least for $d$ small enough) temperature, the behavior of the polymer path is super-diffusive with transversal fluctuations of order $n^{1 / 2+\epsilon}$ for some $\epsilon>0$, while in infinite

2000 Mathematics Subject Classification. 60G57,60G70,82D60.

Key words and phrases. Directed Polymers, Last Passage Percolation, Heavy Tails, Regular Variation. 
$(d \leqslant 2)$ or high enough $(d>2)$ temperature the polymer is diffusive with fluctuations of order $n^{1 / 2}$. These two phases - the strong-disorder phase and weak-disorder phase (respectively) and the conditions for their occurrence should be universal with respect to a large class of environment distributions, including the case where $\left\{\sigma(\{z\}): z \in \mathbb{Z}_{+} \times \mathbb{Z}^{d}\right\}$ are i.i.d. with distribution whose tail decays sufficiently fast. Partial results in this direction have been established in $[2,3,8,14]$.

In the strong disorder phase the attraction to regions in the environment with relatively low energy has a non-negligible counter-effect to the entropic tendency of the polymer to diffuse, resulting in a pinning or localization of the polymer to a region in the environment where the balance between entropy and energy is optimal. The transversal fluctuations of the polymer are therefore significantly effected by the fluctuations in the shape of this favorable region. This pinning becomes absolute in the extreme case of zero temperature (formally, the weak limit of $\mu_{\beta}^{\sigma}$ as $\beta \rightarrow \infty$ ). Here entropy no longer plays a part and the system is uniformly in one of its ground states - states in which the polymer path is a minimizer of the energy $\mathcal{H}^{\sigma}(\cdot)$ (equivalently, maximizer of $\sigma(\cdot)$ ). Universal super-diffusive behavior is expected here as well and some progress has been made in this direction (see $[1,9,11,13]$ and also the surveys on the equivalent models of Directed First/Last Passage Percolation in $[10,12])$.

In this paper we address the case where $(\sigma(\{z\}))_{z}$ are i.i.d. but their distribution has a (right) tail which is heavy enough to fall outside the universality classes discussed above. Inspired by [6], we assume that the tail of the distribution at each site is regularly varying with index $\alpha \in(0,2)$, namely

$$
\mathbb{P}(\sigma(\{z\})>t)=t^{-\alpha} L(t),
$$

where $L(t)$ is a slowly varying function $(L(t u) / L(t) \rightarrow 1$ as $t \rightarrow \infty$ for all $u>0$ - this, of course, includes all constants). We also assume that $\sigma(\{z\})$ are positive absolutelycontinuous random variables and, for the sake of simplicitly, treat only the $d=1$ case. We show [Theorem 2.1] that for any finite temperature, the polymer is localized to the path along which the energy is minimal, i.e. thermal fluctuations are negligible - this is because entropy is of smaller order compared to energy in this case. Consequently, it is natural to let $\beta$ go to zero with $n$ and indeed if

$$
\beta_{n}=\beta n^{1-2 / \alpha} L_{0}(n)
$$

where $L_{0}(n)$ is a related slowly varying function, the system exhibits a non-trivial interplay between energy and entropy. Under this scaling, we show [Theorem 2.1] that the $n$ monomer polymer chain is localized (in probability, exponentially fast) to a cylindrical region of diameter $o(n)$ around a random favorable curve [Equation (2.7)], i.e. one that optimally balances entropy and energy under $\sigma$. Zero (resp. infinite) temperature behavior is recovered if $\beta_{n}$ grows faster (resp. slower) than $n^{1-2 / \alpha} L_{0}(n)$.

A weak limit for the distribution of the favorable curve under linear scaling is then shown to exist [Theorem 2.2] and the limiting distribution (on a proper topological space of directed curves lying in the $45^{\circ}$-rotated square with unit diagonal) is explicitly described. The limit is constructed as the distribution of the (almost-surely) unique global solution to a variational problem on the space of curves [Equation (2.9)]. The functional being maximized is random and can be viewed as assigning to a curve the difference between its 
entropy gain and energy cost under a proper (random) limit environment. These limiting distributions form a two-parameter family of measures on curves $\left\{\mathbb{M}_{\alpha, \beta}, \alpha \in(0,2), \beta \in\right.$ $[0, \infty]\}$ [Proposition 2.4], where $\mathbb{M}_{\alpha, \infty}$ is the scaling limit of the ground-state path, which was studied in [6].

Combining these results we obtain [Corollary 2.3] a linear scaling limit for the unconditional distribution of the polymer's path and transversal fluctuations of order $n$ - quite different from the light tail case. This happens for all $\beta>0$ (under scaling (1.3)). Nevertheless [Proposition 2.5], when $\alpha$ is small enough there exists a random variable $\beta_{c}$ positive a.s. (but arbitrarily small with positive probability) such that if $\beta<\beta_{c}$ then the polymer localizes around the $x$-axis $(s \equiv 0)$, thereby exhibiting an infinite-temperature behavior and if $\beta>\beta_{c}$, the polymer tends to drift away from the axis. This can be viewed as a quenched phase transition with a random threshold value.

Although we only treat the $1+1$ dimensional case here, the problem is exactly the same for all dimensions, with minor changes due to the different geometry. In particular, for any $d$, the right normalization is $\beta_{n}=\beta n^{1-(d+1) / \alpha} L_{0}(n)$ and the limit curves live inside a $d+1$ regular polyhedron.

\section{Setup And Results}

In this section we introduce notation and state our main results. Let $n$ be a positive natural number. Set $\mathbb{L}=\{a(1,1)+b(1,-1): a, b \in \mathbb{Z}\}$ and $\mathbb{L}_{n}=\frac{1}{n} \mathbb{L}$. Define $\mathcal{D}=\{(x, y)$ : $|y| \leqslant \min (x, 1-x)\}, \mathcal{D}^{0}=\mathcal{D} \backslash\{(0,0),(1,0)\}$ and $\mathcal{D}_{n}, \mathcal{D}_{n}^{0}$ as the intersection of $\mathcal{D}, \mathcal{D}^{0}$ with $\mathbb{L}_{n}$. Let $\mathcal{L}^{0}$ be the set of all real Lipschitz functions on $[0,1]$ with Lipschitz constant 1 , vanishing at 0 and 1 and

$$
\mathcal{L}_{n}^{0}=\left\{s \in \mathcal{L}^{0}:(k / n, s(k / n)) \in \mathbb{L}_{n}, k=0, \ldots, n\right\} .
$$

$\mathcal{L}_{n}^{0}$ can be viewed as the set of linearly interpolated $1 / n$-scaled trajectories of a simple random walk conditioned to hit 0 at time $n$ and hence a finite subset of $\mathcal{L}^{0}$. Note that $\gamma \in \mathcal{L}^{0} \Rightarrow \operatorname{graph}(\gamma) \subseteq \mathcal{D}$. Endow $\mathcal{L}^{0}, \mathcal{L}_{n}^{0}$ with the $L^{\infty}$ norm and Borel sigma algebras $\mathcal{B}^{0}$ and $\mathcal{B}_{n}^{0}$. Let $\mathcal{P}$ (resp. $\mathcal{P}_{n}$ ) be the set of all probability measures on $\mathcal{L}^{0}, \mathcal{B}^{0}$ (resp. $\mathcal{L}_{n}^{0}, \mathcal{B}_{n}^{0}$ ). We shall treat $\mathcal{P}_{n}$ as a subset of $\mathcal{P}$.

The entropy of a $\gamma \in \mathcal{L}^{0}$ curve is $-E(\gamma)$ where

$$
E(\gamma)=\int_{0}^{1} e\left(\gamma^{\prime}(x)\right) d x
$$

and $e:[-1,1] \rightarrow \mathbb{R}$ is defined as

$$
e(x)=\frac{1}{2}[(1+x) \log (1+x)+(1-x) \log (1-x)] .
$$

$E$ is well defined since $\gamma$ is differentiable almost everywhere with $\left|\gamma^{\prime}(x)\right| \leqslant 1$. In fact, it is the rate function in the large deviations principle for the sequence of uniform measures on $\mathcal{L}_{n}^{0}$ (this is essentially Mogulskii's Theorem - see [4], Section 5.1).

$\sigma_{n}$ will denote the $\frac{1}{n}$-scaled and $\mathcal{D}_{n}^{0}$-restricted version of $\sigma$. That is, $\sigma_{n}$ is a positive measure on $\mathcal{D}_{n}^{0}$ with i.i.d weights which satisfy (1.2). The scaled analog of $\mu_{\beta}^{\sigma}$ is

$$
\mu_{n, \beta}(s)=\frac{1}{Q_{n, \beta}} \exp \left(\beta \sigma_{n}(s)\right) \quad \text { for } s \in \mathcal{L}_{n}^{0} .
$$


It is a standard fact (see Section 1.1 in [15]) that the distribution of $\sigma_{n}(\{z\})$ is in the max-domain of attraction of the Fréchet distribution, namely there exist $\left(b_{n}\right)_{n} \geqslant 1$ such that if $\left(U_{n}^{i}, Z_{n}^{i}\right) \in\left(\mathbb{R}_{+}, \mathcal{D}_{n}^{0}\right)$ are the value and position of the non-ascending $i$-th order statistic of $\left(\sigma_{n}(\{z\})\right)_{z}$, then for any fixed $k \geqslant 1$

$$
\left(\left(b_{n}^{-1} U_{n}^{i}, Z_{n}^{i}\right)\right)_{i=1}^{k} \Rightarrow\left(\left(V^{i}, Z^{i}\right)\right)_{i=1}^{k},
$$

as $n \rightarrow \infty$, where the limit is non-degenerate. The constants $b_{n}$ can be written as

$$
b_{n}=n^{2 / \alpha} L_{0}(n)
$$

where $L_{0}(n)$ is a related slowly varying function. This is the motivation behind the scaling of the temperature and accordingly if $\beta_{n}$ denotes inverse temperature at system order $n$, we assume

$$
\lim _{n \rightarrow \infty} \frac{b_{n}}{n} \beta_{n}=\bar{\beta}_{\infty}
$$

where $\bar{\beta}_{\infty} \in[0, \infty]=[0, \infty) \cup\{\infty\}$. This is a more explicit version of $(1.3)$.

The favorable curve, around which concentration occurs, is $\gamma_{n, \beta_{n}}^{*}$, where

$$
\gamma_{n, \beta}^{*}=\underset{\gamma \in \mathcal{L}^{0}}{\arg \max }\left(\beta \sigma_{n}(\gamma)-n E(\gamma)\right) .
$$

The properness of this definition is discussed in Lemma 4.1. Localization is "exponentially fast in probability", by which we mean

Theorem 2.1. For all $\epsilon>0, \delta>0$ there exist $\nu>0$ such that

$$
\begin{array}{ll}
\mu_{n, \beta_{n}}\left(\left\|s-\gamma_{n, \beta_{n}}^{*}\right\|_{\infty}>\delta\right) \leqslant e^{-n \nu} & \text { if } \bar{\beta}_{\infty}<\infty, \\
\mu_{n, \beta_{n}}\left(\left\|s-\gamma_{n, \beta_{n}}^{*}\right\|_{\infty}>\delta\right) \leqslant e^{-\nu b_{n} \beta_{n}} & \text { if } \bar{\beta}_{\infty}=\infty,
\end{array}
$$

with $\mathbb{P}_{n}$-probability at least $1-\epsilon$ as long as $n$ is large enough.

Denote by $\mathbb{M}_{n, \beta} \in \mathcal{P}$ the distribution of $\gamma_{n, \beta}^{*}$, namely $\mathbb{M}_{n, \beta}(\cdot)=\mathbb{P}_{n}\left(\gamma_{n, \beta}^{*} \in \cdot\right)$. Under (2.6) the sequence of measures $\left(\mathbb{M}_{n, \beta_{n}}\right)_{n \geqslant 1}$ has a weak limit in $\mathcal{P}$. This limit measure is constructed on top of the scaling limit of the position and weight of the environment point masses, i.e. the infinite collection $\left(\left(V^{i}, Z^{i}\right)\right)_{i=1}^{\infty}$ of which each finite subset $\left(\left(V^{i}, Z^{i}\right)\right)_{i=1}^{k}$ has a law as in the limit in (2.4). It is a standard fact that $\left(Z^{i}\right)_{i=1}^{\infty},\left(V^{i}\right)_{i=1}^{\infty}$ are independent of each other and

$$
Z^{i} \sim \operatorname{Uniform}(\mathcal{D}) \quad \text { i.i.d. } \quad ; \quad V^{i} \stackrel{d}{=} T_{i}^{-\frac{1}{\alpha}}
$$

where $T_{i}$ is the sum of $i$ independent exponentials with rate 1 . We can then define the "limit environment" as

$$
\pi_{\infty}=\sum_{i} V^{i} \delta\left(\cdot-Z^{i}\right) .
$$

Note that while $\pi_{\infty}$ as a measure may be infinite (for $\alpha \geqslant 1$ ), as a function $\pi_{\infty}(\gamma)=$ $\pi_{\infty}(\operatorname{graph}(\gamma))$, it is bounded on $\mathcal{L}^{0}$, with $\mathbb{P}_{\infty}$-probability 1 , where we denote by $\mathbb{P}_{\infty}$ the underlying measure. This follows from Theorem 2.1 in [6]. 
The limit curve $\hat{\gamma}_{\infty, \beta}$ is defined, analogously to the finite case, as the solution to a variational problem on $\mathcal{L}^{0}$, namely

$$
\hat{\gamma}_{\infty, \beta}= \begin{cases}\arg \max _{\gamma \in \mathcal{L}^{0}}\left(\beta \pi_{\infty}(\gamma)-E(\gamma)\right) & \beta<\infty \\ \arg \max _{\gamma \in \mathcal{L}^{0}} \pi_{\infty}(\gamma) & \beta=\infty\end{cases}
$$

A maximizer always exists and it is unique. This will be proved in Lemma 4.1. Formally, however, we shall set $\hat{\gamma}_{\infty, \beta} \equiv \infty$, if one of these conditions fails. With $\mathbb{M}_{\alpha, \beta}(\cdot)=\mathbb{P}_{\infty}\left(\hat{\gamma}_{\infty, \beta} \in\right.$ $\cdot)$, we can now state

Theorem 2.2. $\mathbb{M}_{n, \beta_{n}} \Rightarrow \mathbb{M}_{\alpha, \bar{\beta}_{\infty}}$ as $n \rightarrow \infty$ in $\mathcal{P}$.

The following is an immediate corollary of Theorems 2.1 and 2.2. Let $S_{n, \beta_{n}}$ be a random variable, taking values in $\mathcal{L}_{n}^{0}$, such that conditioned on the environment $\sigma_{n}$, its distribution is $\mu_{n, \beta_{n}}$. The unconditional distribution of $S_{n, \beta_{n}}$, which we will denote by $\mathbb{Q}_{n, \beta_{n}}$ is obtained by averaging over the environment, namely $\mathbb{Q}_{n, \beta_{n}}=\mathbb{P}_{n} \mu_{n, \beta_{n}}$. Then

Corollary 2.3. $\mathbb{Q}_{n, \beta_{n}} \Rightarrow \mathbb{M}_{\alpha, \bar{\beta}_{\infty}}$ as $n \rightarrow \infty$ in $\mathcal{P}$.

In order to justify that these localization results indeed imply a qualitative $\Theta(n)$ change in the shape of the polymer, we have to argue, in addition, that with positive $\mathbb{P}_{\infty^{-}}$ probability $\hat{\gamma}_{\infty, \beta} \not \equiv 0$. This is included in the next proposition. For two random variables $X, Y$ we write $X \supsetneqq s Y$ if $X \leqslant_{s} Y$ but not $X \stackrel{d}{=} Y$.

Proposition 2.4. If $0 \leqslant \beta_{2}<\beta_{1} \leqslant \infty$ then $E\left(\hat{\gamma}_{\infty, \beta_{2}}\right) \supsetneqq s E\left(\hat{\gamma}_{\infty, \beta_{1}}\right)$, where $\hat{\gamma}_{\infty, \beta_{i}}$ is distributed according to $\mathbb{M}_{\alpha, \beta_{i}}$. In particular $\mathbb{M}_{\alpha, \beta_{1}} \neq \mathbb{M}_{\alpha, \beta_{2}}$.

Hence, $\mathbb{M}_{\alpha, \beta}$ for $\beta \in(0, \infty)$ is different from both $\mathbb{M}_{\alpha, 0}=\delta_{0}$ - the Dirac-mass on the zero function $0 \in \mathcal{L}^{0}$ and $\mathbb{M}_{\alpha, \infty}$ - the distribution of the last passage path, i.e. the path along which the sum of the mass weights of $\pi_{\infty}$ (thought of as passage times) is maximal. The latter was studied in [6] as $P^{*}$.

Nevertheless, it is still quite possible that there exists a non-degenerate random $\pi_{\infty^{-}}$ dependent $\beta_{c}$ such that $\hat{\gamma}_{\infty, \beta} \equiv 0$ if $\beta<\beta_{c}$, but $\hat{\gamma}_{\infty, \beta} \not \equiv 0$ if $\beta>\beta_{c}$. This will show a (random) phase-transition-like phenomenon, where depending on whether the temperature is below or above a random threshold, the effect of the environment is microscopic or macroscopic, measured on the scale of $\Theta(n)$. To make this precise, let us define

$$
\widehat{w}_{\infty, \beta}=\max _{\gamma \in \mathcal{L}^{0}}\left(\beta \pi_{\infty}(\gamma)-E(\gamma)\right) \quad \text { and } \quad \beta_{c}=\inf \left\{\beta \geqslant 0: \widehat{w}_{\infty, \beta}>0\right\}
$$

The following proposition shows that this indeed occurs for $\alpha$ small enough. The restrictions on $\alpha$ are not sharp.

\section{Proposition 2.5.}

(1) $\beta_{c}$ is well defined $\mathbb{P}_{\infty}$-a.s.

(2) $\hat{\gamma}_{\infty, \beta} \equiv 0$ if $\beta<\beta_{c}$ and $\hat{\gamma}_{\infty, \beta} \not \equiv 0$ if $\beta>\beta_{c}$.

(3) if $\alpha \in\left[\frac{1}{2}, 2\right)$ then $\beta_{c}=0$ with $\mathbb{P}_{\infty}$-probability 1 .

(4) if $\alpha \in\left(0, \frac{1}{3}\right)$ then $\beta_{c}>0$ with $\mathbb{P}_{\infty}$-probability 1 . 
2.1. Organization of the paper. In the remainder of the text, we prove the results in this section. Section 3 contains some preliminary definitions and tools, on which we base our proofs. Section 4 contains proofs for Theorems 2.1,2.2 and Corollary 2.3. Section 5 contains proofs for Propositions 2.4 and 2.5. Finally, Section 6 contains proofs for some of the results in Section 3, which we deferred.

\section{Preliminaries}

3.1. Environments and $\mathcal{L}^{0}$ Curves. We shall call an environment any positive (possibly infinite) measure on $\mathcal{D}^{0}$ with countable support, for which the collection of mass weights can be ordered in non-ascending order. If $\sigma$ is an environment, we denote by $\left(v_{\sigma}^{i}, z_{\sigma}^{i}=\right.$ $\left.\left(x_{\sigma}^{i}, y_{\sigma}^{i}\right)\right) \in \mathbb{R}_{+} \times \mathcal{D}^{0}$ the position and weight of the $i$-th mass in this order (if there are masses with equal weight, we suppose that they come with a prescribed order). To the collection $\left\{\left(v_{\sigma}^{i}, z_{\sigma}^{i}\right)\right\}_{i=1}^{|\sigma|}$, where $|\sigma|$ is the cardinality of the support of $\sigma$, we add two pairs $\left(v_{\sigma}^{0}, z_{\sigma}^{0}\right),\left(v_{\sigma}^{\infty}, z_{\sigma}^{\infty}\right)$ with $v_{\sigma}^{0}=v_{\sigma}^{\infty}=0$ and $z_{\sigma}^{0}=(0,0), z_{\sigma}^{\infty}=(1,0)$. This will simplify things later on. Thus, $\sigma$ is identified with two sequences:

$$
\left.\left.v_{\sigma}=\left(v_{\sigma}^{i}\right): i=0,1, \ldots,|\sigma|, \infty\right), \quad z_{\sigma}=\left(z_{\sigma}^{i}\right): i=0,1, \ldots,|\sigma|, \infty\right)
$$

and

$$
\sigma=\sum_{i=0,1, \ldots,|\sigma|, \infty} v_{\sigma}^{i} \delta\left(\cdot-z_{\sigma}^{i}\right)
$$

Both $\sigma_{n}$ and $\pi_{\infty}$ in Section 2 are environments under this definition.

Given $i \neq j \in\{0,1, \ldots,|\sigma|, \infty\}$, let $\Delta x_{\sigma}(i, j)=x_{\sigma}^{j}-x_{\sigma}^{i}, \Delta y_{\sigma}(i, j)=y_{\sigma}^{j}-y_{\sigma}^{i}, a_{\sigma}(i, j)=$ $\Delta y_{\sigma}(i, j) / \Delta x_{\sigma}(i, j)$ and set

$$
\mathcal{I}(\sigma)=\left\{\iota \subseteq\{0,1, \ldots,|\sigma|, \infty\}: \quad 0, \infty \in \iota,\left|a_{\sigma}(i, j)\right| \leqslant 1 \forall i \neq j \in \iota\right\} .
$$

If $\iota \in \mathcal{I}(\sigma)$ is finite, we shall also treat it as a sequence of indices $\iota=\left(\iota_{j}\right)_{1 \leqslant j \leqslant|\iota|}$ ordered according to the $x$-coordinate of the indexed point (i.e. $\left.j<k \Rightarrow x_{\sigma}^{\iota_{j}}<x_{\sigma}^{\iota_{k}}\right) . m(\sigma)$ will denote the "mesh" of $\sigma$, defined as

$$
m(\sigma)=\inf _{i \neq j}\left(\left|\Delta x_{\sigma}(i, j)\right| \wedge|| a_{\sigma}(i, j)|-1|\right),
$$

where the infimum is over all $i, j \in\{0,1, \ldots,|\sigma|, \infty\}$. The distance between two environments $\sigma, \widetilde{\sigma}$ with equal cardinality $|\sigma|=|\widetilde{\sigma}|=\chi \leqslant \infty$ is given by

$$
d(\sigma, \widetilde{\sigma})=d_{\chi}(\sigma, \widetilde{\sigma})=\left\|v_{\sigma}-v_{\widetilde{\sigma}}\right\|_{\infty} \vee\left\|z_{\sigma}-z_{\widetilde{\sigma}}\right\|_{\infty} .
$$

This is a well-defined metric on $\Sigma_{\chi}$ - the space of all environments with cardinality $\chi$. We shall also use $\Sigma_{\chi, n} \subseteq \Sigma_{\chi}$ for the subset of environments supported on $\mathcal{D}_{n}^{0}$ and $\Sigma_{\chi}\left(m_{0}\right) \subseteq$ $\Sigma_{\chi}$ for the subset of environments $\sigma$ with $m(\sigma)>m_{0}$. The intersection is denoted by $\Sigma_{\chi, n}\left(m_{0}\right)$.

Given $\phi: \mathcal{X} \rightarrow \mathbb{R}$, where $\mathcal{X}$ is a closed subset of $[0,1]$ with $L=\min (\mathcal{X})$ and $R=$ $\max (\mathcal{X})$, we define linear $(\phi)$ as the $[L, R] \rightarrow \mathbb{R}$ function obtained from $\phi$ by linearly interpolating inside all intervals $\{[l(x), r(x)]: x \notin \mathcal{X}\}$, where $l(x)=\max (\mathcal{X} \cap[0, x])$ and $r(x)=\min (\mathcal{X} \cap[x, 1])$. Given $\mathcal{Z} \subseteq \mathcal{D}$, which is a graph of such function $\phi_{\mathcal{Z}}$, we set linear $(\mathcal{Z})=$ linear $\left(\phi_{\mathcal{Z}}\right)$. 
The following two mappings between $\mathcal{L}^{0}$ and $\mathcal{I}(\sigma)$ will be used often in what follows. $I_{\sigma}: \mathcal{L}^{0} \rightarrow \mathcal{I}(\sigma)$ is defined as

$$
I(\gamma)=\left\{j \in\{0,1, \ldots,|\sigma|, \infty\}: z_{\sigma}^{j} \in \operatorname{graph}(\gamma)\right\} \quad \text { for } \gamma \in \mathcal{L}^{0},
$$

and $\Gamma_{\sigma}: \mathcal{I}(\sigma) \rightarrow \mathcal{L}^{0}$ as

$$
\Gamma_{\sigma}(\iota)=\operatorname{linear}\left(\overline{\left\{z_{\sigma}^{j}: j \in \iota\right\}}\right) \quad \text { for } \iota \in \mathcal{I}(\sigma) .
$$

The validity of these definitions is not difficult to verify. Finally, define $\Lambda_{\sigma}: \mathcal{L}^{0} \rightarrow \mathcal{L}^{0}$ as

$$
\Lambda_{\sigma}(\gamma)=\Gamma\left(I_{\sigma}(\gamma)\right) \quad \text { for } \gamma \in \mathcal{L}^{0}
$$

Below are three technical propositions which we use later in the text. We defer their proofs to Section 6. Recall the definition of $E$ in (2.1).

\section{Proposition 3.1.}

(1) $E$ is lower semi-continuous, strictly convex and positive away from 0.

(2) If $\mathcal{Z}$ is a closed subset of graph $(\gamma)$ for $\gamma \in \mathcal{L}^{0}$, then $E(\operatorname{linear}(\mathcal{Z})) \leqslant E(\gamma)$. In particular, $E\left(\Lambda_{\sigma}(\gamma)\right) \leqslant E(\gamma)$ for any $\gamma \in \mathcal{L}^{0}$ and any environment $\sigma$.

\section{Proposition 3.2.}

(1) For all $\chi_{0}<\infty, \sigma \in \Sigma_{\chi_{0}}$ there exists $d_{0}=d_{0}(\sigma)>0$ such that $\mathcal{I}(\tilde{\sigma}) \subseteq \mathcal{I}(\sigma)$ for all $\tilde{\sigma} \in \Sigma_{\chi_{0}}$ with $d(\sigma, \tilde{\sigma})<d_{0}$. If in addition $m(\sigma)>0$, then the same holds with equality in place of inclusion.

(2) $m(\cdot)$ is uniformly continuous on $\Sigma_{\chi_{0}}\left(m_{0}\right)$ for all $m_{0}>0, \chi_{0}<\infty$.

(3) For all $\epsilon>0, m_{0}>0, \chi_{0}<\infty$ there exists $d_{0}=d_{0}\left(\chi_{0}, m_{0}, \epsilon\right)>0$ such that if $\sigma$, $\tilde{\sigma} \in \Sigma_{\chi_{0}}\left(m_{0}\right)$ satisfy $d(\sigma, \tilde{\sigma})<d_{0}$ then for all $\gamma \in \mathcal{L}^{0}$ there exists $\tilde{\gamma} \in \mathcal{L}^{0}$ such that

(a) $\|\gamma-\tilde{\gamma}\|_{\infty}<\epsilon$.

(b) $\tilde{\sigma}(\widetilde{\gamma})>\sigma(\gamma)-\epsilon$.

(c) $E(\tilde{\gamma})<E(\gamma)+\epsilon$.

The following are well-known results about large deviation of simple random walk paths. The emphasis is on the uniformity of the statements. We define $\mu_{n} \in \mathcal{P}_{n}$ as the uniform measure on $\mathcal{L}_{n}^{0}$.

\section{Proposition 3.3.}

(1) For any fixed $m_{0}>0, \chi_{0}<\infty$ as $n \rightarrow \infty$

$$
-\frac{1}{n} \log \mu_{n}\left(\iota \subseteq I_{\sigma}(s)\right)=E\left(\Gamma_{\sigma}(\iota)\right)+o(1),
$$

uniformly in all $\sigma \in \Sigma_{\chi_{0}, n}\left(m_{0}\right)$ and $\iota \in \mathcal{I}(\sigma)$.

(2) For all $\delta>0$, there exists $\eta=\eta(\delta)>0$ such that as $n \rightarrow \infty$,

$$
-\frac{1}{n} \log \mu_{n}\left(\left\|s-\Gamma_{\sigma}(\iota)\right\|_{\infty}>\delta \mid I_{\sigma}(s)=\iota\right) \geqslant \eta+o(1)
$$

uniformly in all $\sigma \in \Sigma_{\chi_{0}, n}\left(m_{0}\right)$ and $\iota \in \mathcal{I}(\sigma)$ once $m_{0}>0$ and $\chi_{0}<\infty$ are fixed. 
3.2. Weight-Scaled Environments, Environment Truncation and $\delta$-Optimality. For stating the results, it was convenient to work with spatially scaled quantities, such as $\sigma_{n}$ and $\mu_{n, \beta}$. For the proofs, it will turn out useful to define versions of these quantities, which are also weight-scaled. For $\beta \in[0, \infty)$ we set $\bar{\beta}=b_{n} n^{-1} \beta$. In place of $\sigma_{n}$ and $\mu_{n, \beta}$ we have

$$
\pi_{n}=b_{n}^{-1} \sigma_{n} \quad, \quad \bar{\mu}_{n, \beta}(s)=\frac{1}{\bar{Q}_{n, \beta}} \exp \left(n \beta \pi_{n}(s)\right) \quad \text { for } s \in \mathcal{L}_{n}^{0} .
$$

Clearly $\mu_{n, \beta}=\bar{\mu}_{n, \bar{\beta}}$ and (2.6) reads as

$$
\bar{\beta}_{n} \rightarrow \bar{\beta}_{\infty} \text { as } n \rightarrow \infty \quad ; \quad \bar{\beta}_{\infty} \in[0, \infty] .
$$

For $n \leqslant \infty, k<\infty$ we define the truncated environment $\pi_{n}^{k}$ as the one obtained from $\pi_{n}$ by removing the masses at indices $i>k$ (recall that masses are ordered in non-ascending order of their weights). Clearly $\pi_{n}, \pi_{n}^{k}, \pi_{\infty}$ are random elements of $\Sigma_{n^{2} / 4-2}, \Sigma_{k}, \Sigma_{\infty}$ respectively and (2.4) can be written as

$$
\pi_{n}^{k} \Rightarrow \pi_{\infty}^{k} \text { as } n \rightarrow \infty \text {. }
$$

The corresponding polymer measure $\bar{\mu}_{n, \beta}^{k}$ and the normalization factor $\bar{Q}_{n, \beta}^{k}$ are defined as in (3.3), but only with $\pi_{n}^{k}$ in place of $\pi_{n}$.

Next, for $1 \leqslant k, n \leqslant \infty, \beta \in[0, \infty]$, we define the "worthiness" $W_{n, \beta}^{k}$ of an $\mathcal{L}^{0}$-path under environment $\pi_{n}^{k}$. If $n=\infty$, we set

$$
W_{\infty, \beta}^{k}(\gamma)= \begin{cases}\beta \pi_{\infty}^{k}(\gamma)-E(\gamma) & \text { if } \beta<\infty \\ \pi_{\infty}^{k}(\gamma) & \text { if } \beta=\infty\end{cases}
$$

and if $n<\infty$

$$
W_{n, \beta}^{k}(\gamma)= \begin{cases}\beta \pi_{n}^{k}(\gamma)-E(\gamma) & \text { if } \bar{\beta}_{\infty}<\infty \text { or } \beta=0 . \\ \pi_{n}^{k}(\gamma)-\frac{1}{\beta} E(\gamma) & \text { if } \bar{\beta}_{\infty}=\infty \text { or } \beta=\infty\end{cases}
$$

We set $\widehat{\gamma}_{n, \beta}^{k}$ and $\widehat{w}_{n, \beta}^{k}$ to be the maximizer of $W_{n, \beta}^{k}$ in $\mathcal{L}^{0}$ and its value. It will be shown in Lemma 4.1 that this is well-defined for all values of $n, \beta, k$, except if $\beta=\infty, n \wedge k<\infty$, in which case a maximizer exist but it is not unique and we apply any a priori deterministic rule for selecting one of the maximizers as $\hat{\gamma}_{n, \infty}^{k}$ (for instance, we may choose the unique one that minimizes $E$ ). The definition of $\hat{\gamma}_{n, \beta}^{k}$ clearly extends both (2.7) and (2.9) with $\hat{\gamma}_{n, \bar{\beta}}^{\infty}=\gamma_{n, \beta}^{*}$ and $\hat{\gamma}_{\infty, \beta}^{\infty}=\hat{\gamma}_{\infty, \beta}$. From now on we shall omit the superscript $k$ if it is $\infty$. If $\delta>0$, we also need

$$
\widehat{w}_{n, \beta}^{k}(\delta)=\max _{\gamma \in \mathcal{L}^{0}:\left\|\gamma-\widehat{\gamma}_{n, \beta}^{k}\right\|_{\infty} \geqslant \delta} W_{n, \beta}^{k}(\gamma) .
$$

Finally, we define the remainder environment as $\rho_{n}^{k}=\pi_{n}-\pi_{n}^{k}$ and its maximal contribution to (minus) the energy of a $\mathcal{L}^{0}$ path is $R_{n}^{k}=\max _{\gamma \in \mathcal{L}^{0}} \rho_{n}^{k}(\gamma)$.

\section{Localization}

We now prove the main localization results, using 4 lemmas which we state in the beginning of this section. The proofs for these lemmas are deferred to the end of this section, and we first prove Theorems 2.1,2.2 and Corollary 2.3. 
The first lemma establishes the existence and uniqueness of $\hat{\gamma}_{n, \beta}^{k}$ and shows that truncated quantities are good approximations. The first part is due to [6] (Lemma 3.1).

\section{Lemma 4.1.}

(1) For all $n \leqslant \infty$ we have $R_{n}^{k} \rightarrow 0$ as $k \rightarrow \infty$ with $\mathbb{P}_{n}$-probability 1 .

(2) For all $1 \leqslant k, n \leqslant \infty, \beta \in[0, \infty]$ and $\delta>0$ quantities $\hat{\gamma}_{n, \beta}^{k}, \widehat{w}_{n, \beta}^{k}$ and $\widehat{w}_{n, \beta}^{k}(\delta)$ are well defined with $\mathbb{P}_{n}$-probability 1 .

(3) For all $\beta \in[0, \infty], n \leqslant \infty$ we have $\hat{\gamma}_{n, \beta}^{k} \rightarrow \hat{\gamma}_{n, \beta}$ as $k \rightarrow \infty$ with $\mathbb{P}_{n}$-probability 1.

(4) for all $\delta>0, \beta \in[0, \infty]$ we have $\liminf _{k \rightarrow \infty} \widehat{w}_{\infty, \beta}^{k}-\widehat{w}_{\infty, \beta}^{k}(\delta)>0$ with $\mathbb{P}_{\infty}$-probability 1 .

In this lemma we show that truncated quantities of both the finite and limiting system can be coupled such that they are arbitrarily close to each other.

Lemma 4.2. For all $\delta>0, \epsilon>0$, there exist $K,\left(N_{k}\right)_{k} \geqslant K$ such that for all $k \geqslant K$ and $n \geqslant N_{k}$ there exists a coupling $\mathbb{P}_{n+\infty}$ of $\pi_{n}^{k}$ and $\pi_{\infty}^{k}$ under which with probability at least $1-\epsilon$ :

(1) $\left|\widehat{w}_{n, \bar{\beta}_{n}}^{k}-\widehat{w}_{\infty, \bar{\beta}}^{k}\right|<\epsilon$

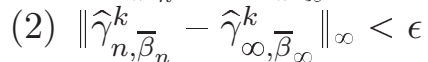

(3) $\widehat{w}_{n, \bar{\beta}_{n}}^{k}(\delta)<\widehat{w}_{\infty, \bar{\beta}_{\infty}}^{k}(\delta / 2)+\epsilon$

The following improves on the results of Lemma 4.1 as it shows that approximation by truncated quantities can be made uniform in $n$. The first part is Proposition 3.3 in [6].

\section{Lemma 4.3.}

(1) For all $\epsilon, \delta>0$ there exists $K$ such that $R_{n}^{k}<\delta$ with $\mathbb{P}_{n}$-probability at least $1-\epsilon$ for all $k>K$ and all $n \leqslant \infty$.

(2) For all $\epsilon, \delta>0$ there exists $K$ such that $\left\|\hat{\gamma}_{n, \bar{\beta}_{n}}^{k}-\widehat{\gamma}_{n, \bar{\beta}_{n}}\right\|_{\infty}<\delta$ with $\mathbb{P}_{n}$-probability at least $1-\epsilon$ for all $k>K$ and all $n \leqslant \infty$.

(3) For all $\epsilon, \delta>0$, there exists $K, \eta>0$ such that $\hat{w}_{n, \bar{\beta}_{n}}^{k}(\delta)<\widehat{w}_{n, \bar{\beta}_{n}}^{k}-\eta$ with $\mathbb{P}_{n^{-}}$ probability at least $1-\epsilon$ for all $k>K$ and $k \leqslant n \leqslant \infty$.

In this lemma we show concentration with truncated quantities (unless $\bar{\beta}_{\infty}=\infty$, in which case this is essentially Theorem 2.1).

Lemma 4.4. For all $\delta>0, \epsilon>0$, there exists $\nu>0$ and $K,\left(N_{k}\right)_{k} \geqslant K\left(\right.$ case $\left.\bar{\beta}_{\infty}<\infty\right)$ or $N$ (case $\left.\bar{\beta}_{\infty}=\infty\right)$ such that with $\mathbb{P}_{n}$-probability at least $1-\epsilon$

$$
\begin{array}{ll}
-\frac{1}{n} \log \bar{\mu}_{n, \bar{\beta}_{n}}^{k}\left(s:\left\|s-\hat{\gamma}_{n, \bar{\beta}_{n}}^{k}\right\|_{\infty}>\delta\right) \geqslant \nu & \left(\text { case } \bar{\beta}_{\infty}<\infty\right) \\
-\frac{1}{n} \log \bar{\mu}_{n, \bar{\beta}_{n}}\left(s:\left\|s-\hat{\gamma}_{n, \bar{\beta}_{n}}\right\|_{\infty}>\delta\right) \geqslant \nu \bar{\beta}_{n} & \left(\text { case } \bar{\beta}_{\infty}=\infty\right),
\end{array}
$$

for all $k>K, n>N_{k}\left(\right.$ case $\left.\bar{\beta}_{\infty}<\infty\right)$ or $n>N\left(\right.$ case $\left.\bar{\beta}_{\infty}=\infty\right)$. 
Proof of Theorem 2.1. If $\bar{\beta}_{\infty}=\infty$ we can just quote Lemma 4.4. Otherwise, fix $\epsilon>0$, $\delta>0$ and write

$$
\begin{aligned}
\bar{\mu}_{n, \bar{\beta}_{n}}\left(s: \| s-\widehat{\gamma}_{\left.n, \bar{\beta}_{n} \|_{\infty}>\delta\right) \leqslant}^{k} \bar{\mu}_{n, \bar{\beta}_{n}}^{k}\left(s:\left\|s-\widehat{\gamma}_{n, \bar{\beta}_{n}}^{k}\right\|_{\infty}>\delta\right)\right. \\
\times \sup \left\{\frac{d \bar{\mu}_{n, \bar{\beta}_{n}}}{\left.d \bar{\mu}_{n, \bar{\beta}_{n}}^{k}(s):\left\|s-\hat{\gamma}_{n, \bar{\beta}_{n}}^{k}\right\|_{\infty}>\delta\right\}}\right.
\end{aligned}
$$

By Lemma 4.4 the first factor on the r.h.s. is exponentially decaying in $n$ with some rate $\nu>0$ with probability at least $1-\epsilon$ for all properly large $k, n$. On the other hand

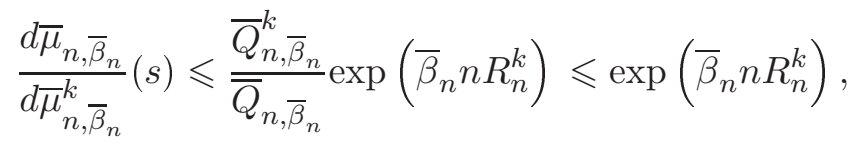

and therefore using Lemma 4.3 part (1) and choosing $k$ large enough, we can have the second factor in (4.2) grow exponentially in $n$ with rate at most $\nu / 2$ also with probability at least $1-\epsilon$. Finally, from part (2) of Lemma 4.3 for possibly larger $k$, we can have also $\left\|\hat{\gamma}_{n, \bar{\beta}_{n}}-\hat{\gamma}_{n, \bar{\beta}_{n}}^{k}\right\|_{\infty}<\delta / 2$ with the same probability. Combining the above, we complete the proof.

Proof of Theorem 2.2. Fix $\epsilon, \delta>0$. From Lemma 4.1, 4.2 and 4.3 it follows that we can find $k$ large enough and then $n$ large enough such that with $\mathbb{P}_{n+\infty}$ probability at least $1-\epsilon$

$$
\left\|\hat{\gamma}_{n, \bar{\beta}_{n}}-\hat{\gamma}_{n, \bar{\beta}_{n}}^{k}\right\|_{\infty}<\delta, \quad\left\|\hat{\gamma}_{n, \bar{\beta}_{n}}^{k}-\hat{\gamma}_{\infty, \bar{\beta}_{\infty}}^{k}\right\|_{\infty}<\delta, \quad\left\|\hat{\gamma}_{\infty, \bar{\beta}_{\infty}}^{k}-\hat{\gamma}_{\infty, \bar{\beta}_{\infty}}\right\|_{\infty}<\delta .
$$

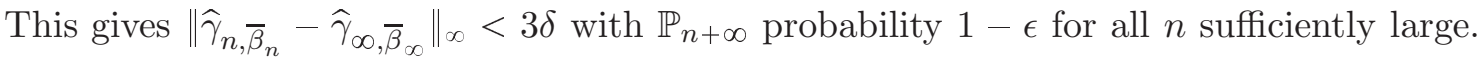
Since $\epsilon, \delta$ are arbitrary, the result follows.

Proof of Corollary 2.3. We can use Skorohod Representation Theorem or the proof of Theorem 2.2 , to conclude that for any $\epsilon, \delta>0$ if $n$ is large enough $\mathbb{P}_{n+\infty}\left(\left\|\hat{\gamma}_{n, \bar{\beta}_{n}}-\hat{\gamma}_{\infty, \bar{\beta}_{\infty}}\right\|_{\infty}<\right.$ $\delta)>1-\epsilon$. Then, possibly for larger $n$, from Theorem 2.1 we have $\bar{\mu}_{n, \bar{\beta}_{n}}\left(\left\|s-\hat{\gamma}_{n, \bar{\beta}_{n}}\right\|_{\infty}>\delta\right) \leqslant \epsilon$ with $\mathbb{P}_{n}$-probability at least $1-\epsilon$. By the total probability formula this implies $\mathbb{P}_{n}\left(\| S_{n, \beta_{n}}-\right.$ $\left.\hat{\gamma}_{n, \bar{\beta}_{n}} \|_{\infty}>\delta\right) \leqslant 2 \epsilon$. All together we have $\mathbb{P}_{n+\infty}\left(\left\|S_{n, \beta_{n}}-\hat{\gamma}_{\infty, \bar{\beta}_{\infty}}\right\|_{\infty}>2 \delta\right) \leqslant 3 \epsilon$ and since $\delta, \epsilon$ are arbitrary, the result follows.

Proof of Lemma 4.1. Part (1) is Lemma 3.1 in [6]. For part (2), existence of a maximizer in (2.9) will follow if we show that $W_{n, \beta}^{k}$ is upper semi-continuous, since $\mathcal{L}^{0}$ is compact in the $\|\cdot\|_{\infty}$ topology. Indeed, $E(\cdot)$ is lower semi-continuous (Proposition 3.1). As for $\pi_{n}^{k}(\cdot)$, from part (1), given $\epsilon>0$ and $\gamma_{0} \in \mathcal{L}^{0}$ we may find $k_{1}$ such that $R_{n}^{k_{1}}<\epsilon$, and set $\delta=\min \left\{\left|y_{\sigma}^{l}-\gamma_{0}\left(x_{\sigma}^{l}\right)\right|: 1 \leqslant l<k_{1}, y_{\sigma}^{l} \neq \gamma_{0}\left(x_{\sigma}^{l}\right)\right\}$. Then, for $\left\|\gamma-\gamma_{0}\right\|_{\infty}<\delta$

$$
\pi_{n}^{k}(\gamma)<\pi_{n}^{k}\left(\gamma_{0}\right)+R_{n}^{k_{1}}<\pi_{n}^{k}\left(\gamma_{0}\right)+\epsilon
$$

which implies upper semi-continuity.

It remains to show uniqueness. If $\beta=0$ we have $\hat{\gamma}_{n, 0}^{k} \equiv 0$, which is the minimum of $E$ (Proposition 3.1). If $\beta=n=k=\infty$, uniqueness was proved in [6] (see Proposition 
4.1 and the unique way to extend $U^{*}$ to a continuous increasing path $\left.P^{*}\right)$. For $\beta=\infty$, $n \wedge k<\infty$ uniqueness holds by definition. In the remaining cases, assume the contrary and let $\hat{\gamma}_{0}, \breve{\gamma}_{0}$ be two different maximizers of $W_{n, \beta}^{k}$. Then $\hat{\iota}_{0}=I_{\pi_{n}^{k}}\left(\hat{\gamma}_{0}\right)$ must be different from $\breve{\iota}_{0}=I_{\pi_{n}^{k}}\left(\breve{\gamma}_{0}\right)$, because the minimizer of $E(\gamma)$ in $\left\{\gamma \in \mathcal{L}^{0}: I_{\pi_{n}^{k}}(\gamma)=\iota\right\}$ is unique, as it follows from the strict convexity of $E(\cdot)$. We proceed as in Proposition 4.1 in [6]. Without loss of generality there must exists $1 \leqslant j \leqslant n$ such that with positive $\mathbb{P}_{n}$-probability

$$
\max _{\gamma: j \in I_{\pi_{n}^{k}}(\gamma)} W_{n, \beta}^{k}(\gamma)=\max _{\gamma: j \notin I_{\pi_{n}^{k}}(\gamma)} W_{n, \beta}^{k}(\gamma) .
$$

But conditioned on $\left(Z_{n}^{i}\right)_{i} \geqslant 1,\left(U_{n}^{i}\right)_{i \neq j}$ the r.h.s of the above event is a constant while the l.h.s is an absolutely continuous (w.r.t. Lebesgue measure) random variable. It follows that this probability is zero, which is a contradiction. This proves part (2).

Part (3) is is trivial if $n<\infty$. If $n=\infty$, by compactness $\exists\left(k_{l}\right)_{l} \geqslant 1, \widetilde{\gamma}_{0} \in \mathcal{L}^{0}$ s.t. $\hat{\gamma}_{\infty, \beta}^{k_{l}} \rightarrow \widetilde{\gamma}_{0}$ as $l \rightarrow \infty$. Then

$$
\begin{aligned}
W_{\infty, \beta}\left(\tilde{\gamma}_{0}\right) & \geqslant \limsup _{l \rightarrow \infty} W_{\infty, \beta}\left(\hat{\gamma}_{\infty, \beta}^{k_{l}}\right) \geqslant \limsup _{l \rightarrow \infty} W_{\infty, \beta}^{k_{l}}\left(\hat{\gamma}_{\infty, \beta}^{k_{l}}\right) \\
& \geqslant \limsup _{l \rightarrow \infty} W_{\infty, \beta}^{k_{l}}\left(\hat{\gamma}_{\infty, \beta}\right)=W_{\infty, \beta}\left(\hat{\gamma}_{\infty, \beta}\right)=\hat{w}_{\infty, \beta},
\end{aligned}
$$

where the first inequality follows from upper semi-continuity. By uniqueness it must be that $\widetilde{\gamma}_{0}=\hat{\gamma}_{\infty, \beta}$ and since this is true for any subsequence of $\hat{\gamma}_{\infty, \beta}^{k}$, the result follows.

As for part (4), if the statement had been false, then there would have been sequences $\left(k_{l}\right)_{l \geqslant 1}$ and $\left(\breve{\gamma}_{\infty, \beta}^{k_{l}}\right)_{l \geqslant 1}$ such that $\lim \sup _{l \rightarrow \infty} W_{\infty, \beta}^{k_{l}}\left(\breve{\gamma}_{\infty, \beta}^{k_{l}}\right) \geqslant \liminf _{l \rightarrow \infty} W_{\infty, \beta}^{k_{l}}\left(\hat{\gamma}_{\infty, \beta}^{k_{l}}\right)$ and $\left\|\breve{\gamma}_{\infty, \beta}^{k_{l}}-\widehat{\gamma}_{\infty, \beta}^{k_{l}}\right\|_{\infty} \geqslant \delta$. By compactness we could further suppose that $\breve{\gamma}_{\infty, \beta}^{k_{l}} \rightarrow \breve{\gamma}_{\infty, \beta}$ as $l \rightarrow \infty$. Then upper semi-continuity would have implied

$$
\begin{aligned}
W_{\infty, \beta}\left(\check{\gamma}_{\infty, \beta}\right) & \geqslant \limsup _{l \rightarrow \infty} W_{\infty, \beta}\left(\breve{\gamma}_{\infty, \beta}^{k_{l}}\right) \geqslant \limsup _{l \rightarrow \infty} W_{\infty, \beta}^{k_{l}}\left(\breve{\gamma}_{\infty, \beta}^{k_{l}}\right) \\
& \geqslant \liminf _{l \rightarrow \infty} W_{\infty, \beta}^{k_{l}}\left(\hat{\gamma}_{\infty, \beta}^{k_{l}}\right) \geqslant \liminf _{l \rightarrow \infty} W_{\infty, \beta}^{k_{l}}\left(\hat{\gamma}_{\infty, \beta}\right)=W_{\infty, \beta}\left(\hat{\gamma}_{\infty, \beta}\right),
\end{aligned}
$$

and part (3) would have given $\left\|\breve{\gamma}_{\infty, \beta}-\hat{\gamma}_{\infty, \beta}\right\|_{\infty} \geqslant \delta>0$. This would have violated the uniqueness of the global maximizer.

Proof of Lemma 4.2. As in Proposition 3.2 of [6], it follows from (3.5) and Skorohod Representation Theorem that for any $k, d_{0}>0$, we can couple together $\pi_{n}^{k}$ and $\pi_{\infty}^{k}$ such that $d\left(\pi_{n}^{k}, \pi_{\infty}^{k}\right)<d_{0}$ with arbitrarily high probability as long as $n$ is large enough. Call this coupling measure $\mathbb{P}_{n+\infty}$ and observe that the absolute continuity of $Z_{\infty}^{j}, j=1, \ldots k$ implies that by choosing $m_{0}$ sufficiently small, we can make $m\left(\pi_{\infty}^{k}\right)>m_{0}$ occur with $\mathbb{P}_{n+\infty}$ probability arbitrarily close to 1 . Using Proposition 3.2 part (2), if $d_{0}$ is chosen small enough, this implies $\pi_{n}^{k}, \pi_{\infty}^{k} \in \Sigma_{k}\left(m_{0} / 2\right)$. Now given $\epsilon>0$, Proposition 3.2 part (3) applied to $\pi_{n}^{k}$ and $\pi_{\infty}^{k}$ with $\widehat{\gamma}_{n, \bar{\beta}_{n}}^{k}$ and $\hat{\gamma}_{\infty, \bar{\beta}_{\infty}}^{k}$ together with the assumption on $\bar{\beta}_{n}$ guarantee that by further restricting $d_{0}$ we have $\left|\widehat{w}_{n, \bar{\beta}_{n}}^{k}-\widehat{w}_{\infty, \bar{\beta}_{\infty}}^{k}\right|<\epsilon$. This shows (1).

As for (2), for any $\epsilon>0$ it follows from Lemma 4.1 part (4), that there exists $\eta>0$, such that $\widehat{w}_{\infty, \bar{\beta}_{\infty}}^{k}(\epsilon)<\widehat{w}_{\infty, \bar{\beta}_{\infty}}^{k}-\eta$, if $k$ is large enough with arbitrarily high $\mathbb{P}_{n+\infty}$ probability. 
Then, using Proposition 3.2 part (3) again, for $d_{0}$ and $n$ large it must be that $W_{n, \bar{\beta}_{n}}^{k}(\gamma)<$ $\widehat{w}_{\infty, \bar{\beta}_{\infty}}^{k}-\eta / 2$ for all $\gamma \in \mathcal{L}^{0}$ such that $\left\|\gamma-\widehat{\gamma}_{\infty, \bar{\beta}_{\infty}}^{k}\right\|_{\infty}>2 \epsilon$. Then from part (1), for possibly smaller $d_{0}$, all such $\gamma$ satisfy $W_{n, \bar{\beta}_{n}}^{k}(\gamma)<\widehat{w}_{n, \bar{\beta}_{n}}^{k}-\eta / 3$. This shows $\left\|\hat{\gamma}_{n, \bar{\beta}_{n}}^{k}-\hat{\gamma}_{\infty, \bar{\beta}_{\infty}}^{k}\right\|_{\infty} \leqslant 2 \epsilon$ as required.

Finally, given $\delta>0$ we may find $\gamma \in \mathcal{L}^{0}$ satisfying $\left\|\gamma-\hat{\gamma}_{n, \bar{\beta}_{n}}^{k}\right\|_{\infty} \geqslant \delta$ and $W_{n, \bar{\beta}_{n}}^{k}(\gamma)=$ $\widehat{w}_{n, \bar{\beta}_{n}}^{k}(\delta)$. Then using part (2) and Proposition 3.2 part (3), provided $d_{0}$ is sufficiently small and $k, n$ are sufficiently large, we may find $\tilde{\gamma} \in \mathcal{L}^{0}$ satisfying $\left\|\widetilde{\gamma}-\widehat{\gamma}_{\infty, \bar{\beta}_{\infty}}^{k}\right\|_{\infty} \geqslant \delta / 2$ and $W_{\infty, \bar{\beta}_{\infty}}^{k}(\widetilde{\gamma})>\widehat{w}_{n, \bar{\beta}_{n}}^{k}(\delta)-\epsilon$ where $\epsilon>0$ is given. This shows $(3)$.

Proof of Lemma 4.3. Part (1) is Proposition 3.3 in [6]. For part (2), fix $\delta, \epsilon>0$ and use Lemma 4.1 part (4) to find $\eta>0$ such that $\widehat{w}_{\infty, \bar{\beta}_{\infty}}^{k}(\delta)<\widehat{w}_{\infty, \bar{\beta}_{\infty}}^{k}-\eta$ with $\mathbb{P}_{\infty}$-probability at least $1-\epsilon$, as long as $k$ is large enough. Then, use Lemma 4.2, to find $n$ large enough such that

$$
\widehat{w}_{n, \bar{\beta}_{n}}^{k}(2 \delta)<\widehat{w}_{n, \bar{\beta}_{n}}^{k}-\eta / 2
$$

with $\mathbb{P}_{n+\infty}$-probability at least $1-2 \epsilon$. Now, from part (1), with probability at least $1-3 \epsilon$ we can also have $R_{n}^{k}<\eta / 4$ for all $n$, possibly by further restricting $k$. In this case, it must be that

$$
\left\|\widehat{\gamma}_{n, \bar{\beta}_{n}}^{l}-\widehat{\gamma}_{n, \bar{\beta}_{n}}^{k}\right\|_{\infty}<2 \delta
$$

for all $l \geqslant k$. Then $\left\|\hat{\gamma}_{n, \bar{\beta}_{n}}^{l}-\hat{\gamma}_{n, \bar{\beta}_{n}}\right\|_{\infty}<4 \delta$ for all $l \geqslant k$. This shows part (2). But then from $(4.3),(4.4)$ and the restriction on $R_{n}^{k}$, we have $\widehat{w}_{n, \bar{\beta}_{n}}^{l}(4 \delta)<\widehat{w}_{n, \bar{\beta}_{n}}^{l}-\eta / 4$ for all $l \geqslant k$, for possibly larger $k$. This shows part (3). The restriction on $n$ can be enforced by a restriction on $k$.

Proof of Lemma 4.4. Let us treat the $\bar{\beta}_{\infty}<\infty$ case first. Write

$$
\begin{aligned}
\bar{\mu}_{n, \bar{\beta}_{n}}^{k}\left(s:\left\|s-\hat{\gamma}_{n, \bar{\beta}_{n}}^{k}\right\|_{\infty}>\delta\right) \\
\leqslant \bar{\mu}_{n, \bar{\beta}_{n}}^{k}\left(s:\left\|\Lambda_{\pi_{n}^{k}}(s)-\hat{\gamma}_{n, \bar{\beta}_{n}}^{k}\right\|_{\infty} \geqslant \delta / 2\right) \\
\quad+\bar{\mu}_{n, \bar{\beta}_{n}}^{k}\left(s:\left\|s-\Lambda_{\pi_{n}^{k}}(s)\right\|_{\infty}>\delta / 2,\left\|\Lambda_{\pi_{n}^{k}}(s)-\hat{\gamma}_{n, \bar{\beta}_{n}}^{k}\right\|_{\infty}<\delta / 2\right) .
\end{aligned}
$$

Now,

$$
\bar{\mu}_{n, \bar{\beta}_{n}}^{k}\left(s:\left\|\Lambda_{\pi_{n}^{k}}(s)-\hat{\gamma}_{n, \bar{\beta}_{n}}^{k}\right\|_{\infty} \geqslant \delta / 2\right) \leqslant \sum_{\iota} \bar{\mu}_{n, \bar{\beta}_{n}}^{k}\left(I_{\pi_{n}^{k}}(s)=\iota\right)
$$

where the sum is over all $\iota \in \mathcal{I}\left(\pi_{n}^{k}\right)$ such that $\left\|\Gamma_{\pi_{n}^{k}}(\iota)-\hat{\gamma}_{n, \bar{\beta}_{n}}^{k}\right\|_{\infty} \geqslant \delta / 2$. Each such term satisfies

$$
\begin{aligned}
\bar{\mu}_{n, \bar{\beta}_{n}}^{k}\left(I_{\pi_{n}^{k}}(s)=\iota\right) & =\frac{\mu_{n}\left[\exp \left(n \bar{\beta}_{n} \pi_{n}^{k}(s)\right) ; I_{\pi_{n}^{k}}(s)=\iota\right]}{\mu_{n}\left[\exp \left(n \bar{\beta}_{n} \pi_{n}^{k}(s)\right)\right]} \\
& \leqslant \frac{\exp \left(n \bar{\beta}_{n} \pi_{n}^{k}\left(\Gamma_{\pi_{n}^{k}}(\iota)\right)\right) \mu_{n}\left(\iota \subseteq I_{\pi_{n}^{k}}(s)\right)}{\exp \left(n \bar{\beta}_{n} \pi_{n}^{k}\left(\hat{\gamma}_{n, \bar{\beta}_{n}}^{k}\right)\right) \mu_{n}\left(I_{\pi_{n}^{k}}\left(\hat{\gamma}_{n, \bar{\beta}_{n}}^{k}\right) \subseteq I_{\pi_{n}^{k}}(s)\right)} .
\end{aligned}
$$


As in the previous proof, for any $k>0$ we may find $m_{0}>0$, such that with arbitrarily high probability $\pi_{n}^{k} \in \Sigma_{k}\left(m_{0}\right)$ as long as $n$ is large enough. Then, by Proposition 3.1 part (2) and Proposition 3.3 part (1)

$$
\begin{aligned}
-\frac{1}{n} \log \left[\bar{\mu}_{n, \bar{\beta}_{n}}^{k}\left(I_{\pi_{n}^{k}}(s)=\iota\right)\right] & \geqslant W_{n, \bar{\beta}_{n}}^{k}\left(\Lambda_{\pi_{n}^{k}}\left(\hat{\gamma}_{n, \bar{\beta}_{n}}^{k}\right)\right)-W_{n, \bar{\beta}_{n}}^{k}\left(\Gamma_{\pi_{n}^{k}}(\iota)\right)+o(1) \\
& \geqslant \widehat{w}_{n, \bar{\beta}_{n}}^{k}-W_{n, \bar{\beta}_{n}}^{k}\left(\Gamma_{\pi_{n}^{k}}(\iota)\right)+o(1) .
\end{aligned}
$$

Plugging this into (4.6), noting that there are at most $2^{k}$ terms in the sum there and using Lemma 4.3 part (3), we infer that there exists $\nu_{1}>0$, such that

$$
\mathbb{P}_{n}\left(-\frac{1}{n} \log \bar{\mu}_{n, \bar{\beta}_{n}}^{k}\left(s:\left\|\Lambda_{\pi_{n}^{k}}(s)-\hat{\gamma}_{n, \bar{\beta}_{n}}^{k}\right\|_{\infty} \geqslant \delta / 2\right) \geqslant \nu_{1}\right) \geqslant 1-\epsilon
$$

for large enough $k, n$. At the same time, the second term in (4.5) is clearly bounded by

$$
\sum_{\iota} \bar{\mu}_{n, \bar{\beta}_{n}}^{k}\left(\left\|s-\Gamma_{\pi_{n}^{k}}(\iota)\right\|_{\infty}>\delta / 2 \mid I_{\pi_{n}^{k}}(s)=\iota\right)=\sum_{\iota} \mu_{n}\left(\left\|s-\Gamma_{\pi_{n}^{k}}(\iota)\right\|_{\infty}>\delta / 2 \mid I_{\pi_{n}^{k}}(s)=\iota\right),
$$

where the sum is over all $\iota \in \mathcal{I}\left(\pi_{n}^{k}\right)$ such that $\left\|\Gamma_{\pi_{n}^{k}}(\iota)-\widehat{\gamma}_{n, \bar{\beta}_{n}}^{k}\right\|_{\infty}<\delta / 2$. We may now use Proposition 3.3 part (2) and the bound on the number of terms, to conclude that there exists $\nu_{2}>0$ such that

$$
\mathbb{P}_{n}\left(-\frac{1}{n} \log \left[\bar{\mu}_{n, \bar{\beta}_{n}}^{k}\left(s:\left\|s-\Lambda_{\pi_{n}^{k}}(s)\right\|_{\infty}>\delta / 2,\left\|\Lambda_{\pi_{n}^{k}}(s)-\hat{\gamma}_{n, \bar{\beta}_{n}}^{k}\right\|_{\infty}<\delta / 2\right)\right] \geqslant \nu_{2}\right) \geqslant 1-\epsilon
$$

This holds uniformly in $k$, but $n$ needs to be large enough. Combining (4.5), (4.8) and (4.9), we complete the $\bar{\beta}_{\infty}<\infty$ case.

If $\bar{\beta}_{\infty}=\infty$ we take $k=\left|\mathcal{D}_{n}^{0}\right|=n^{2} / 4-2$. Then from (4.7) we get for all $\gamma \in \mathcal{L}_{n}^{0}$

$$
-\frac{1}{n} \log \bar{\mu}_{n, \bar{\beta}_{n}}(\gamma) \geqslant \bar{\beta}_{n}\left(\widehat{w}_{n, \bar{\beta}_{n}}-W_{n, \bar{\beta}_{n}}(\gamma)\right)+O(1)
$$

Then, since there are at most $2^{n}$ paths and using Lemma 4.3 part (3), we obtain for some $\nu>0$

$$
-\frac{1}{n} \log \bar{\mu}_{n, \bar{\beta}_{n}}\left(s:\left\|s-\widehat{\gamma}_{n, \bar{\beta}_{n}}\right\|_{\infty}>\delta\right) \geqslant \nu \bar{\beta}_{n}-O(1) \geqslant \frac{1}{2} \nu \bar{\beta}_{n},
$$

with $\mathbb{P}_{n}$-probability at least $1-\epsilon$ as long as $n$ is large enough.

\section{The Limit Distribution}

Proof of Proposition 2.4. For $\beta \in(0, \infty)$, set $Y_{\beta}(\gamma)=\beta^{-1} W_{\infty, \beta}(\gamma)=\pi_{\infty}(\gamma)-\beta^{-1} E(\gamma)$. If $0<\beta_{2}<\beta_{1}<\infty$ then

$$
E\left(\hat{\gamma}_{\infty, \beta_{2}}\right)=\frac{Y_{\beta_{1}}\left(\hat{\gamma}_{\infty, \beta_{2}}\right)-Y_{\beta_{2}}\left(\hat{\gamma}_{\infty, \beta_{2}}\right)}{\beta_{2}^{-1}-\beta_{1}^{-1}} \leqslant \frac{Y_{\beta_{1}}\left(\hat{\gamma}_{\infty, \beta_{1}}\right)-Y_{\beta_{2}}\left(\hat{\gamma}_{\infty, \beta_{1}}\right)}{\beta_{2}^{-1}-\beta_{1}^{-1}}=E\left(\hat{\gamma}_{\infty, \beta_{1}}\right) .
$$

This shows $E\left(\hat{\gamma}_{\infty, \beta_{2}}\right) \leqslant s E\left(\hat{\gamma}_{\infty, \beta_{1}}\right)$. It remains to show that $E\left(\hat{\gamma}_{\infty, \beta_{2}}\right)<E\left(\hat{\gamma}_{\infty, \beta_{1}}\right)$ with positive probability. Recall the definitions of $Z^{i}, V^{i}$ and $T_{i}$ in (2.8). For any $\delta>0$, from Lemma 4.1 part (1) we may find $k_{0}$ such that $R_{\infty}^{k_{0}}<\delta$ with $\mathbb{P}_{\infty}$-probability at least $\frac{1}{2}$. Also, there exists $t_{0}$ large enough such that $T_{k_{0}}<t_{0}$ with $\mathbb{P}_{\infty}$-probability at least $\frac{3}{4}$. Then

$$
\frac{1}{2} \leqslant \mathbb{P}_{\infty}\left(R_{\infty}^{k_{0}}<\delta\right)=\mathbb{E}_{\infty}\left(\mathbb{P}_{\infty}\left(R_{\infty}^{k_{0}}<\delta \mid T_{k_{0}}\right)\right) \leqslant \mathbb{P}_{\infty}\left(R_{\infty}^{k_{0}}<\delta \mid T_{k_{0}}=t_{0}\right)+\frac{1}{4}
$$


where for the last inequality we use the obvious monotonicity of $t \mapsto \mathbb{P}_{\infty}\left(R_{\infty}^{k_{0}}<\delta \mid T_{k_{0}}=t\right)$. Since the last term is independent of $k_{0}$ and using the monotonicity again we in fact have

$$
\mathbb{P}_{\infty}\left(R_{\infty}^{k}<\delta \mid T_{k} \geqslant t_{0}\right) \geqslant \frac{1}{4}
$$

for all $k$. Next, it is not difficult to verify that we can find $\delta, \eta>0$ small enough as well as $0<t_{1}<t_{2}<t_{3}$ and $z_{1}, z_{2} \in \mathcal{D}^{0}$, such that on the event

$$
\mathcal{A}=\left\{\left|T_{i}-t_{i}\right|<\eta,\left|Z^{i}-z_{i}\right|<\eta \text { for } i=1,2, T_{3} \geqslant t_{3} \text { and } R_{\infty}^{3}<\delta\right\}
$$

the following holds:

(1) $\left|a_{\pi_{\infty}}(1,2)\right|>1$. In other words, no $\mathcal{L}^{0}$-curve can take both $Z^{1}$ and $Z^{2}$.

(2) $E\left(\gamma_{1}\right)>E\left(\gamma_{2}\right)$ and $W_{\infty, \beta_{i}}\left(\gamma_{j}\right)>0$ for $i, j=1,2$, where $\gamma_{i}=\Gamma_{\pi_{\infty}}(\{0, i, \infty\})$.

(3) $\exists \epsilon>0$ such that $W_{\infty, \beta_{1}}\left(\gamma_{1}\right)-W_{\infty, \beta_{1}}\left(\gamma_{2}\right)>\epsilon$ and $W_{\infty, \beta_{2}}\left(\gamma_{2}\right)-W_{\infty, \beta_{2}}\left(\gamma_{1}\right)>\epsilon$.

(4) $\beta_{1}\left(V^{3}+R_{\infty}^{3}\right)<\epsilon$.

In light of (2.8) and (5.1), event $\mathcal{A}$ has positive probability under $\mathbb{P}_{\infty}$ for any choice of parameters. At the same time, the above conditions guarantee that $\hat{\gamma}_{\infty, \beta_{1}} \neq \hat{\gamma}_{\infty, \beta_{2}}$. Now, if $E\left(\hat{\gamma}_{\infty, \beta_{1}}\right)=E\left(\hat{\gamma}_{\infty, \beta_{2}}\right)$ it must be that $\pi_{\infty}\left(\hat{\gamma}_{\infty, \beta_{1}}\right)=\pi_{\infty}\left(\hat{\gamma}_{\infty, \beta_{2}}\right)$, for otherwise one of $\hat{\gamma}_{\infty, \beta_{i}}$ cannot be a maximizer. But then it has to be the case that $\hat{\gamma}_{\infty, \beta_{1}}, \hat{\gamma}_{\infty, \beta_{2}}$ are both maximizers of $W_{\infty, \beta_{1}}$ and $W_{\infty, \beta_{2}}$ which contradicts uniqueness. Therefore $E\left(\hat{\gamma}_{\infty, \beta_{1}}\right)>E\left(\hat{\gamma}_{\infty, \beta_{2}}\right)$ as desired. The cases $\beta_{2}=0$ and/or $\beta_{1}=\infty$ are proved in a similar way.

Proof of Proposition 2.5. Although $\hat{\gamma}_{\infty, \beta}$ might not be defined for all $\beta$ for a given environment, it is the case for $\widehat{w}_{\infty, \beta}$ with $\mathbb{P}_{\infty}$-probability 1 , as the proof of Lemma 4.1 shows. This makes $\beta_{c}$ well defined and shows part (1). Part (2) holds since if for some $\beta_{0}$ and $\gamma_{0} \in \mathcal{L}^{0} \backslash\{0\}$ we have $W_{\infty, \beta_{0}}\left(\gamma_{0}\right) \geqslant 0$ then $\widehat{w}_{\infty, \beta}>0$ for all $\beta>\beta_{0}$. For parts (3) and (4), define $\gamma_{z} \in \mathcal{L}^{0}$ as the curve $\gamma_{z}=\operatorname{linear}\{(0,0), z,(1,0)\}$, where $z=(x, y) \in \mathcal{D}$. We claim that

$$
C_{1}\left(\frac{y^{2}}{x}+\frac{y^{2}}{1-x}\right) \leqslant E\left(\gamma_{z}\right) \leqslant C_{2}\left(\frac{y^{2}}{x}+\frac{y^{2}}{1-x}\right)
$$

for some positive $C_{1}, C_{2}$. This can be verified by a simple calculation. This in turn implies that the set $\left\{z \in \mathcal{D}: E\left(\gamma_{z}\right) \leqslant \delta\right\}$ has Lebesgue measure $\Theta(\sqrt{\delta})$ as $\delta \rightarrow 0$.

Next, from LLN there exists a.s. $k_{0}$ such that $T_{k} \leqslant 2 k$ for all $k>k_{0}$. Then, conditioning on $\left(T_{k}\right)_{k} \geqslant 1$, for all such $k \geqslant k_{0}$ and any $\beta>0$ we have

$$
\mathbb{P}_{\infty}\left(W_{\infty, \beta}\left(\gamma_{Z^{k}}\right)>0 \mid\left(T_{k}\right)_{k \geqslant 1}\right) \geqslant \mathbb{P}_{\infty}\left(E\left(\gamma_{Z^{k}}\right)<\beta(2 k)^{-\frac{1}{\alpha}}\right) \geqslant C \beta^{1 / 2} k^{-\frac{1}{2 \alpha}}
$$

and events $\left\{W_{\infty, \beta}\left(\gamma_{Z^{k}}\right)>0\right\}_{k} \geqslant k_{0}$ are (conditionally) independent. Now, for $\alpha>\frac{1}{2}$ and any $\beta>0$, the sum of the probabilities above diverges, whence we may conclude via Borel-Cantelli Lemma that with $\mathbb{P}_{\infty}$-probability 1 there will be $k_{1}>k_{0}$ for which $0<$ $W_{\infty, \beta}\left(\gamma_{Z^{k_{1}}}\right) \leqslant \widehat{w}_{\infty, \beta}$. Since $\beta$ is arbitrary the proof for part $(3)$ is complete.

For part (4), we need to show $\mathbb{P}_{\infty}\left(\widehat{w}_{\infty, \beta}=0\right) \rightarrow 1$ as $\beta \rightarrow 0$. Let $\epsilon>0$ be arbitrarily small. It is not difficult to see that there exists $\eta>0$ such that $T_{k} \geqslant \eta k$ for all $k \geqslant 1$ with $\mathbb{P}_{\infty}$-probability at least $1-\epsilon$. On this event and if $\alpha<1$

$$
Q^{k} \triangleq \sum_{m \geqslant k} V^{m} \leqslant C k^{-\left(\frac{1}{\alpha}-1\right)} \text {. }
$$


Now, for $\gamma \in \mathcal{L}^{0}$ set $l(\gamma)=\min \left(I_{\pi_{\infty}}(\gamma) \backslash\{0\}\right)$ - the smallest index of a mass reached by $\gamma$. Then Proposition 3.1-(2) implies that $W_{\infty, \beta}(\gamma) \leqslant \beta Q^{l(\gamma)}-E\left(\gamma_{Z^{l(\gamma)}}\right)$ for all $\gamma \in \mathcal{L}^{0}$. Therefore, if $\alpha$ is further restricted $\alpha<\frac{1}{3}$ and $\beta$ is small enough,

$$
\begin{aligned}
\mathbb{P}_{\infty}\left(\widehat{w}_{\infty, \beta}=0\right)+\epsilon & \geqslant \mathbb{P}_{\infty}\left(\beta Q^{k}-E\left(\gamma_{Z^{k}}\right) \leqslant 0 \text { for all } k \mid T_{k} \geqslant \eta k ; k=1, \ldots\right) \\
& \geqslant \prod_{k=1}^{\infty}\left(1-C \beta^{1 / 2} k^{-\left(\frac{1}{2 \alpha}-\frac{1}{2}\right)}\right) \\
& \geqslant \exp \left\{-C \beta^{1 / 2} \sum_{k=1}^{\infty} k^{-\left(\frac{1}{2 \alpha}-\frac{1}{2}\right)}\right\}
\end{aligned}
$$

and the last term goes to 1 as $\beta \rightarrow 0$. Since $\epsilon$ is arbitrarily, this concludes the last part of the proposition.

\section{Proofs for Subsection 3.1}

Proof of Proposition 3.1. E is lower semi-continuous as a rate function of a large deviations principle. Strict convexity and positivity away from 0 are inherited from $e$. This shows (1). As for (2), by Jensen's inequality for any $0 \leqslant l<r \leqslant 1$ we have

$$
\int_{l}^{r} e\left(\gamma^{\prime}(x)\right) d x \geqslant(r-l) e\left(\frac{1}{r-l} \int_{l}^{r} \gamma^{\prime}(x) d x\right)=(r-l) e\left(\frac{\gamma(r)-\gamma(l)}{r-l}\right) .
$$

This gives $E\left(\operatorname{linear}(\{(l, \gamma(l)),(r, \gamma(r))\}) \mathbb{I}_{[l, r]}\right) \leqslant E\left(\gamma \mathbb{1}_{[l, r]}\right)$ and the proof is completed by summation.

Proof of Proposition 3.2. The first two parts are easy to verify. As for the third, fix $\chi_{0}<\infty, m_{0}>0, \gamma \in \mathcal{L}^{0}$, let $\sigma, \tilde{\sigma} \in \Sigma_{\chi_{0}}\left(m_{0}\right)$ and set $d_{0}=d(\sigma, \tilde{\sigma})$. We shall show that, once $d_{0}$ is small enough, we can explicitly construct a $\widetilde{\gamma} \in \mathcal{L}^{0}$ satisfying (3a), (3b), (3c) with $\epsilon=\epsilon\left(\chi_{0}, m_{0}, d_{0}\right)$ independently of $\gamma, \sigma, \widetilde{\sigma}$ and that $\epsilon\left(\chi_{0}, m_{0}, d_{0}\right) \rightarrow 0$ as $d_{0} \rightarrow 0$ for all $\chi_{0}, m_{0}$. In what follows, we shall often omit the $\sigma, \widetilde{\sigma}$ subscript and instead add tilde above quantities related to $\widetilde{\sigma}$. Let $\iota=\left(\iota_{j}\right)_{j=1}^{|\iota|}=I_{\sigma}(\gamma)$ and recall that the indices in $\iota$ are ordered according to the $x$-coordinate of the indexed masses. This induces a piecewise decomposition of $\gamma$ :

$$
\gamma=\sum_{j=1}^{|\iota|-1} \gamma^{j} \quad ; \quad \gamma^{j}=\gamma \mathbb{I}_{\left[x^{\iota_{j}}, x^{\iota j+1}\right)} .
$$

We will use this decomposition to construct $\widetilde{\gamma}$. Formally for $j=1, \ldots,|\iota|-1$ set

$$
\widetilde{\gamma}_{ \pm}^{j}(x)=\widetilde{y}^{\iota_{j}}+a_{ \pm}^{j}\left(\gamma^{j}\left(x^{\iota_{j}}+b^{j}\left(x-\widetilde{x}^{\iota_{j}}\right)\right)-y^{\iota_{j}}\right)+c_{ \pm}^{j}\left(x-\widetilde{x}^{\iota_{j}}\right) \mathbb{I}_{\left[\widetilde{x}^{\iota_{j}, \widetilde{x}^{\iota_{j}+1}}\right)},
$$

where

$$
a_{ \pm}^{j}=\frac{\Delta \widetilde{x}\left(\iota_{j}, \iota_{j+1}\right) \mp \Delta \widetilde{y}\left(\iota_{j}, \iota_{j+1}\right)}{\Delta x\left(\iota_{j}, \iota_{j+1}\right) \mp \Delta y\left(\iota_{j}, \iota_{j+1}\right)} ; \quad b^{j}=\frac{\Delta x\left(\iota_{j}, \iota_{j+1}\right)}{\Delta \widetilde{x}\left(\iota_{j}, \iota_{j+1}\right)} ; \quad c_{ \pm}^{j}= \pm\left(1-a_{ \pm}^{j} b^{j}\right),
$$

and let $\tilde{\gamma}=\sum_{j=1}^{|\iota|-1} \tilde{\gamma}^{j}$ where

$$
\tilde{\gamma}^{j}= \begin{cases}\tilde{\gamma}_{+}^{j} & \text { if } \tilde{a}\left(\iota_{j}, \iota_{j+1}\right) \geqslant a\left(\iota_{j}, \iota_{j+1}\right) . \\ \widetilde{\gamma}_{-}^{j} & \text { otherwise. }\end{cases}
$$


We now argue that $\widetilde{\gamma}$ is in $\mathcal{L}^{0}$ and satisfies (3a), (3b), (3c). Indeed, it is easy to verify that each piece $\widetilde{\gamma}^{j}$ is supported on $\left[\widetilde{x}^{\iota_{j}}, \widetilde{x}^{\iota_{j+1}}\right)$ and satisfies $\widetilde{\gamma}^{j}\left(\widetilde{x}^{\iota_{j}}\right)=\widetilde{y}^{\iota_{j}}$ and $\widetilde{\gamma}^{j}\left(\left(\widetilde{x}^{\iota_{j+1}}\right)^{-}\right)=$ $\widetilde{y}^{\iota_{j+1}}$. Also not difficult is $a_{ \pm}^{j}=1+o(1), b^{j}=1+o(1), c_{ \pm}^{j}=o(1)$ as $d_{0} \rightarrow 0$ and

$$
c_{ \pm}^{j}=\frac{\widetilde{a}\left(\iota_{j}, \iota_{j+1}\right)-a\left(\iota_{j}, \iota_{j+1}\right)}{1 \mp a\left(\iota_{j}, \iota_{j+1}\right)}
$$

which implies that $\pm c_{ \pm}^{j} \geqslant 0$ when it is used for $\widetilde{\gamma}^{j}$ in (6.1). Then since $\frac{d}{d x} \widetilde{\gamma}_{ \pm}^{j}=\frac{d}{d x} \gamma^{j}+$ $c_{ \pm}^{j}\left(1 \mp \frac{d}{d x} \gamma^{j}\right)$ and $\left|\frac{d}{d x} \gamma^{j}\right| \leqslant 1$ on $\left(x^{\iota_{j}}, x^{\iota_{j+1}}\right)$, it follows that $\left|\frac{d}{d x} \widetilde{\gamma}_{ \pm}^{j}\right| \leqslant 1$ on $\left(\widetilde{x}^{\iota_{j}}, \tilde{x}^{\iota_{j+1}}\right)$ once $d_{0}$ is sufficiently small. This shows that $\tilde{\gamma} \in \mathcal{L}^{0}$.

Now, since $\gamma, \tilde{\gamma} \in \mathcal{L}^{0}$, we have for all $j$ and $x \in\left[x^{\iota_{j}} \vee \widetilde{x}^{\iota_{j}}, x^{\iota_{j+1}} \wedge \widetilde{x}^{\iota_{j+1}}\right)$

$$
\begin{aligned}
\left|\gamma^{j}(x)-\widetilde{\gamma}^{j}(x)\right| & =\left|\widetilde{y}^{\iota_{j}}+a_{ \pm}^{j}\left(\gamma^{j}\left(x^{\iota_{j}}+b^{j}\left(x-\widetilde{x}^{\iota_{j}}\right)\right)-y^{\iota_{j}}\right)+c_{ \pm}^{j}\left(x-\widetilde{x}^{\iota_{j}}\right)-\gamma^{j}(x)\right| \\
& =\left|\widetilde{y}^{\iota_{j}}+\gamma^{j}(x)-y^{\iota_{j}}-\gamma^{j}(x)+o(1)\right|=o(1) .
\end{aligned}
$$

as $d_{0} \rightarrow 0$. Therefore (3a) follows from

$$
\|\gamma-\widetilde{\gamma}\|_{\infty} \leqslant\left(\sup _{1 \leqslant j \leqslant|\iota|-1}\left\|\left(\gamma^{j}-\widetilde{\gamma}^{j}\right) \mathbb{I}_{\left[x^{\iota_{j}} \vee \widetilde{x}^{\iota_{j}}, x^{\iota_{j}+1} \wedge \widetilde{x}^{\iota_{j}+1}\right)}\right\|_{\infty}\right)+2 \sup _{1 \leqslant j \leqslant|\iota|}\left|x^{\iota_{j}}-\widetilde{x}^{\iota_{j}}\right|=o(1) .
$$

For (3b) write

$$
\widetilde{\sigma}(\widetilde{\gamma}) \geqslant \sum_{j=1}^{|\iota|} \widetilde{v}^{\iota_{j}} \geqslant \sum_{j=1}^{|\iota|}\left(v^{\iota_{j}}+o(1)\right)=\sigma(\gamma)+o(1) .
$$

Finally (3c) comes from

$$
\begin{aligned}
E(\widetilde{\gamma}) & =\int_{0}^{1} e\left(\frac{d}{d x} \widetilde{\gamma}\right) d x=\sum_{j=1}^{|\iota|-1} \int_{\widetilde{x}^{\iota j}}^{\widetilde{x}^{\iota j} j+1} e\left(\frac{d}{d x} \widetilde{\gamma}\right) d x=\sum_{j=1}^{|\iota|-1} \int_{\widetilde{x}^{\iota j}}^{\widetilde{x}^{\iota j}+1} e\left(\frac{d}{d x} \gamma+o(1)\right) d x \\
& \leqslant \sum_{j=1}^{|\iota|-1} \frac{1}{b^{j}} \int_{x^{\iota j}}^{x^{\iota j} j+1} e\left(\frac{d}{d x} \gamma\right) d x+o(1)=E(\gamma)+o(1),
\end{aligned}
$$

where we used the uniform continuity and boundedness of $e$ in $[-1,1]$.

Proof of Proposition 3.3. We shall use a standard tilting argument. Let $\mu$ be a probability measure under which $\left(\xi_{i}\right)_{i=1}^{\infty}$ are independent, symmetric \pm 1 random variables. Set $S_{0}=0$, $S_{k}=\sum_{i=1}^{k} \xi_{i}$ for $k=1, \ldots$ For any $\lambda \in \mathbb{R}$, we denote by $\mu^{\lambda}$ the exponential tilting of $\mu$, namely

$$
\mu^{\lambda}\left(\xi_{i}=x\right)=\frac{e^{\lambda x} \mu(\xi=x)}{\mu e^{\lambda \xi_{i}}}
$$

where $\mu f$ denotes expectation of $f$ with respect to $\mu$.

It is easy to verify that

$$
\mu^{\lambda}\left(S_{n}=x\right)=\exp \{\lambda x-n L(\lambda)\} \mu\left(S_{n}=x\right),
$$

where $L(\lambda)=\log \left(\mu e^{\lambda \xi_{i}}\right)$. Moreover, all moments of $\xi_{i}$ under $\mu^{\lambda}$ are $C^{\infty}$ as a function of $\lambda$ and in particular $\lambda \mapsto \mu^{\lambda} \xi_{i}=L^{\prime}(\lambda)$ is increasing and tending to \pm 1 as $\lambda \rightarrow \pm \infty$. 
If $|x|<n$, we may choose $\lambda=\lambda(x)$ such that $\mu^{\lambda} \xi_{i}=L^{\prime}(\lambda(x))=\frac{x}{n}$, in which case $e\left(\frac{x}{n}\right)=\lambda(x) \frac{x}{n}-L(\lambda(x))$ where $e(x)=\sup _{\lambda \in \mathbb{R}}\{\lambda x-L(\lambda)\}$ and the latter is an implicit form of (2.2) (see, for instance, Lemma 2.2.5 in [4]). Then (6.2) becomes

$$
\mu\left(S_{n}=x\right)=\exp \left\{-n e\left(\frac{x}{n}\right)\right\} \mu^{\lambda(x)}\left(S_{n}=x\right) .
$$

We may then use local Central Limit Theorem for $S_{n}$ under $\mu^{\lambda}$, which holds uniformly on any bounded set of $\lambda$-s, since in this case we have a uniform bound on moments of $\xi_{i}$. It follows that for any $\theta<1$, there exists $C_{1}=C_{1}(\theta)>0, C_{2}=C_{2}(\theta)>0$ such that

$$
C_{1} \exp \left\{-n e\left(\frac{x}{n}\right)\right\} n^{-1 / 2} \leqslant \mu\left(S_{n}=x\right) \leqslant C_{2} \exp \left\{-n e\left(\frac{x}{n}\right)\right\} n^{-1 / 2}
$$

for all $x$ such that $\mu\left(S_{n}=x\right) \neq 0$ and $\left|\frac{x}{n}\right|<\theta$. This implies

$$
-\frac{1}{n} \log \mu\left(S_{n}=x\right)=e\left(\frac{x}{n}\right)+o(1) .
$$

Now fix $\chi_{0}<\infty, m_{0}>0$ and let $\sigma \in \Sigma_{\chi_{0}, n}\left(m_{0}\right)$ and $\iota \in \mathcal{I}(\sigma)$. Then

$$
\begin{aligned}
-\frac{1}{n} \log \mu_{n}\left(\iota \subseteq I_{\sigma}(s)\right) & =-\frac{1}{n} \sum_{j=1}^{|\iota|-1} \log \mu\left(S_{n \Delta x_{\sigma}\left(\iota_{j}, \iota_{j+1}\right)}=n \Delta y_{\sigma}\left(\iota_{j}, \iota_{j+1}\right)\right) \\
& =\sum_{j=1}^{|\iota|-1} \Delta x_{\sigma}\left(\iota_{j}, \iota_{j+1}\right)\left(e\left(a_{\sigma}\left(\iota_{j}, \iota_{j+1}\right)\right)+o(1)\right) \\
& =E\left(\Gamma_{\sigma}(\iota)\right)+o(1),
\end{aligned}
$$

uniformly as desired.

As for the second part, fix in addition $\delta>0$ and let $\sigma, \iota$ be as before. Then

$$
\begin{aligned}
& \mu_{n}\left(\left\|s-\Gamma_{\sigma}(\iota)\right\|_{\infty}>\delta \mid I_{\sigma}(s)=\iota\right) \\
& \leqslant \sum_{j=1}^{|\iota|-1} \mu\left(\exists 0 \leqslant k \leqslant n \Delta x_{\sigma}\left(\iota_{j}, \iota_{j+1}\right):\left|S_{k}-k a_{\sigma}\left(\iota_{j}, \iota_{j+1}\right)\right|>\delta n \mid\right. \\
& \left.\quad S_{n \Delta x_{\sigma}\left(\iota_{j}, \iota_{j+1}\right)}=n \Delta y_{\sigma}\left(\iota_{j}, \iota_{j+1}\right), S_{n \Delta x_{\sigma}\left(\iota_{j}, i\right)} \neq n \Delta y_{\sigma}\left(\iota_{j}, i\right) ; \forall i \notin \iota\right) .
\end{aligned}
$$

For the rest of the proof, we write $\Delta x_{j}, \Delta y_{j}, \Delta a_{j}$ as a short for $\Delta x_{\sigma}\left(\iota_{j}, \iota_{j+1}\right), \Delta y_{\sigma}\left(\iota_{j}, \iota_{j+1}\right)$, $\Delta a_{\sigma}\left(\iota_{j}, \iota_{j+1}\right)$ and $\Delta x_{j}(i), \Delta y_{j}(i)$ as a short for $\Delta x_{\sigma}\left(\iota_{j}, i\right), \Delta y_{\sigma}\left(\iota_{j}, i\right)$. Choosing $\lambda_{j}$ such that $\mu^{\lambda_{j}} \xi_{i}=L^{\prime}\left(\lambda_{j}\right)=a_{j}$ and setting $\widetilde{S}_{k}=S_{k}-k a_{j}$, the $j$-th term in the above sum is equal to

$$
\begin{gathered}
\mu^{\lambda_{j}}\left(\exists \delta n / 2 \leqslant k \leqslant n \Delta x_{j}:\left|\widetilde{S}_{k}\right|>\delta n \mid \widetilde{S}_{n \Delta x_{j}}=0, \widetilde{S}_{n \Delta x_{j}(i)} \neq n\left(\Delta y_{j}(i)-\Delta x_{j}(i) a_{j}\right) ; \forall i \notin \iota\right) \\
\leqslant \frac{\sum_{k=\delta n / 2}^{n \Delta x_{j}} \mu^{\lambda_{j}}\left(\left|\widetilde{S}_{k}\right|>\delta n\right)}{\mu^{\lambda_{j}}\left(\widetilde{S}_{n \Delta x_{j}}=0\right)-\sum_{i \notin \iota} \mu^{\lambda_{j}}\left(\widetilde{S}_{n \Delta x_{j}(i)}=n\left(\Delta y_{j}(i)-\Delta x_{j}(i) a_{j}\right), \widetilde{S}_{n \Delta x_{j}}=0\right)} .
\end{gathered}
$$

For the numerator, Cramer's Theorem implies

$$
-\frac{1}{n} \log \mu^{\lambda_{j}}\left(\left|\widetilde{S}_{k}\right|>\delta n\right) \geqslant\left(\widetilde{e}^{\lambda_{j}}(\delta) \wedge \widetilde{e}^{\lambda_{j}}(-\delta)\right)+o(1),
$$


as $n \rightarrow \infty$, uniformly in the range of $k$, where $\widetilde{e}^{\lambda}(\cdot)$ is Cramer's rate function for $\xi_{i}-\mu^{\lambda} \xi_{i}$ under $\mu^{\lambda}$. It is easy to verify that $\tilde{e}(x) \triangleq \inf _{\lambda \in(-\infty,+\infty)} \tilde{e}^{\lambda}(x)$ is positive away from 0 , whence there exists $\eta=\eta(\delta)$ such that

$$
-\frac{1}{n} \log \left(\sum_{\delta n / 2}^{n \Delta x_{j}} \mu^{\lambda_{j}}\left(\left|\widetilde{S}_{k}\right|>\delta n\right)\right) \geqslant \eta+o(1),
$$

as $n \rightarrow \infty$ uniformly as desired. On the other hand, by local CLT, we have

$$
\mu^{\lambda_{j}}\left(\widetilde{S}_{n \Delta x_{j}}=0\right)=\Omega\left(n^{-1 / 2}\right)
$$

and

$$
\mu^{\lambda_{j}}\left(\widetilde{S}_{n \Delta x_{j}(i)}=n\left(\Delta y_{j}(i)-\Delta x_{j}(i) a_{j}\right), \widetilde{S}_{n \Delta x_{j}}=0\right)=O\left(n^{-1}\right),
$$

as $n \rightarrow \infty$ uniformly in $\sigma \in \Sigma_{\chi_{0}}\left(m_{0}\right), \iota \in \mathcal{I}(\sigma)$ and $j$. Together, this implies that the denominator is $\Omega\left(n^{-1 / 2}\right)$ and the proof is complete.

6.1. Acknowledgements. We would like to thank Gérard Ben Arous for showing us the paper [6] and for fruitful discussions. For the latter we would also like to thank Chuck Newman. The research of both authors was supported in part by NSF Grant OISE0730136. The research of the first author was also supported in part by NSF Grant DMS 0806180.

\section{REFERENCES}

[1] Baik; J.; Deift; P.;McLaughlin; K.T.-R.;Miller; P.; Zhou; X. Optimal tail estimates for directed last passage site percolation with geometric random variables. Adv. Theor. Math. Phys. 5, 1207-1250 (2001).

[2] Bolthausen, E. A note on diffusion of directed polymers in a random environment. Commun. Math. Phys. 123, 529-534 (1989).

[3] Comets F.; Shiga, T. and Yoshida, N., Probabilistic analysis of directed polymers in a random environment: a review. Stochastic analysis on large scale interacting systems, 115-142, Adv. Stud. Pure Math., 39, Math. Soc. Japan, Tokyo, (2004).

[4] Dembo, A.; Zeitouni, O., Large deviations techniques and applications Springer (1998).

[5] Fisher, D. S.; Huse, D. A. Directed paths in random potential., Phys. Rev. B, 43, 10728-10742 (1991).

[6] Hambly, B.; Martin, J. B. Heavy tails in last-passage percolation., Probab. Theory Related Fields 137, no. 1-2, 227-275 (2007).

[7] Huse, D.A.; and Henley, C.L. Pinning and roughening of domain walls in Ising systems due to random impurities. Phys. Rev. Lett. 54, 2708-2711 (1985).

[8] Imbrie, J.Z.; Spencer, T. Diffusion of directed polymer in a random environment. J. Stat. Phys. 52, Nos 3/4, 609-626 (1988).

[9] Johansson, K. Shape fluctuations and random matrices, Commun. Math. Phys., 209, 437-476 (2000).

[10] Kesten, H. Aspects of first passage percolation. École d'Éte de probabilités de Saint-Flour, XIV-1984, 125-264, Lecture Notes in Math., 1180, Springer, Berlin, (1986).

[11] Licea, C.; Newman, C. M.; Piza, M. S. T. Superdiffusivity in first-passage percolation. Probab. Theory Related Fields 106, no. 4, 559-591, (1996).

[12] Martin, J. B. Last-passage percolation with general weight distribution. Markov Processes and Related Fields, vol. 12, pp. 273-299 (2006).

[13] Newman, C.; Piza, M. Divergence of shape fluctuations in two dimensions. Ann. Probab. 23, No. 3, 977-1005 (1995).

[14] Piza, M.S.T. Directed polymers in a random environment: some results on fluctuations. J. Statist. Phys. 89 , no. 3-4, 581-603 (1997). 
[15] Resnick, R. Extreme values, regular variation and point processes. New York [etc.] : Springer, cop. (1987).

A. Auffinger, Courant Institute of the Mathematical Sciences, New York University, 251 Mercer Street, New York, NY 10012, USA

E-mail address: auffing@cims.nyu.edu

O. Louidor, Courant Institute of the Mathematical Sciences, New York University, 251 Mercer Street, New York, NY 10012, USA

E-mail address: louidor@cims.nyu.edu 